\title{
A note on approximate fixed point property and Du-Karapinar-Shahzad's intersection theorems
}

\section{Wei-Shih Du*}

\section{"Correspondence:}

wsdu@nknucc.nknu.edu.tw

Department of Mathematics,

National Kaohsiung Normal

University, Kaohsiung, 824, Taiwan

\begin{abstract}
In this note, we give new short proofs of Du-Karapinar-Shahzad's intersection theorems for multivalued non-self-maps in complete metric spaces.

MSC: $47 \mathrm{H} 10 ; 54 \mathrm{H} 25$
\end{abstract}

Keywords: $\tau$-function; $\mathcal{M T}$-function ( $\mathcal{R}$-function); coincidence point; fixed point; approximate fixed point property

\section{Introduction and preliminaries}

Let us begin with some basic definitions and notations that will be needed in this paper. Let $(X, d)$ be a metric space. Denote by $\mathcal{N}(X)$ the family of all nonempty subsets of $X$ and by $\mathcal{C B}(X)$ the family of all nonempty closed and bounded subsets of $X$. For each $x \in X$ and $A \subseteq X$, let $d(x, A)=\inf _{y \in A} d(x, y)$. A function $\mathcal{H}: \mathcal{C B}(X) \times \mathcal{C B}(X) \rightarrow[0, \infty)$ defined by

$$
\mathcal{H}(A, B)=\max \left\{\sup _{x \in B} d(x, A), \sup _{x \in A} d(x, B)\right\}
$$

is said to be the Hausdorff metric on $\mathcal{C B}(X)$ induced by the metric $d$ on $X$. The symbols $\mathbb{N}$ and $\mathbb{R}$ are used to denote the sets of positive integers and real numbers, respectively.

Let $K$ be a nonempty subset of $X, g: K \rightarrow X$ be a single-valued non-self-map and $T$ : $K \rightarrow \mathcal{N}(X)$ be a multivalued non-self-map. A point $v$ in $X$ is a coincidence point (see, for instance, [1-6]) of $g$ and $T$ if $g v \in T x$. If $g=$ id is the identity map, then $v=g v \in T v$ and call $v$ a fixed point of $T$. The set of fixed points of $T$ and the set of coincidence points of $g$ and $T$ are denoted by $\mathcal{F}_{K}(T)$ and $\mathcal{C O} \mathcal{P}_{K}(g, T)$, respectively. In particular, if $K \equiv X$, we use $\mathcal{F}(T)$ and $\mathcal{C O P}(g, T)$ instead of $\mathcal{F}_{K}(T)$ and $\mathcal{C O P}_{K}(g, T)$, respectively. The map $T$ is said to have approximate fixed point property [1-5] on $K$ provided $\inf _{x \in K} d(x, T x)=0$. It is obvious that $\mathcal{F}_{K}(T) \neq \emptyset$ implies that $T$ has approximate fixed point property.

A function $\varphi:[0, \infty) \rightarrow[0,1)$ is said to be an $\mathcal{M T}$-function (or $\mathcal{R}$-function) [3-11] if $\lim \sup _{s \rightarrow t^{+}} \varphi(s)<1$ for all $t \in[0, \infty)$. Clearly, if $\varphi:[0, \infty) \rightarrow[0,1)$ is a nondecreasing function or a nonincreasing function, then $\varphi$ is an $\mathcal{M T}$-function. So, the set of $\mathcal{M T}$-functions is a rich class and has the questions many of which are worth studying.

The study of fixed points for single-valued non-self-maps or multivalued non-self-maps satisfying certain contractive conditions is an interesting and important direction of research in metric fixed point theory. A great deal of such research has been investigated by

\section{Springer}

๑2013 Du; licensee Springer. This is an Open Access article distributed under the terms of the Creative Commons Attribution License (http://creativecommons.org/licenses/by/2.0), which permits unrestricted use, distribution, and reproduction in any medium, provided the original work is properly cited. 
several authors, see, e.g., [11-19] and the references therein. Very recently, Du, Karapinar and Shahzad [11] established the following intersection existence theorem of coincidence points and fixed points of multivalued non-self-maps of Kannan type and Chatterjea type.

Theorem 1.1 [11, Theorem 8] Let $(X, d)$ be a complete metric space, $K$ be a nonempty closed subset of $X, T: K \rightarrow \mathcal{C B}(X)$ be a multivalued map and $g: K \rightarrow X$ be a continuous map. Suppose that

(D1) $T x \cap K \neq \emptyset$ for all $x \in K$,

(D2) $T x \cap K$ is g-invariant (i.e., $g(T x \cap K) \subseteq T x \cap K)$ for each $x \in K$,

(D3) there exist a function $h: K \rightarrow[0, \infty)$ and $\gamma \in\left[0, \frac{1}{2}\right)$ such that

$$
\begin{aligned}
\mathcal{H}(T x, T y \cap K) \leq & \gamma[d(x, T x \cap K)+d(y, T x \cap K)+d(y, T y \cap K)] \\
& +h(y) d(g y, T x \cap K) \quad \text { for all } x, y \in K .
\end{aligned}
$$

Then $\mathcal{C O P}_{K}(g, T) \cap \mathcal{F}_{K}(T) \neq \emptyset$.

In [11], they also gave some coincidence and fixed point theorems for multivalued nonself-maps of Mizoguchi-Takahashi type, Berinde-Berinde type and Du type.

Theorem 1.2 [11, Theorem 19] Let $(X, d)$ be a complete metric space, $K$ be a nonempty closed subset of $X, T: K \rightarrow \mathcal{C B}(X)$ be a multivalued map and $g: K \rightarrow X$ be a continuous map. Suppose that conditions (D1) and (D2) as in Theorem 1.1 hold. If there exist an $\mathcal{M T}$ function $\varphi:[0, \infty) \rightarrow[0,1)$ and a function $h: K \rightarrow[0, \infty)$ such that

$$
\mathcal{H}(T x, T y \cap K) \leq \varphi(d(x, y)) d(x, y)+h(y) d(g y, T x \cap K) \quad \text { for all } x, y \in K
$$

then $\mathcal{C O P}_{K}(g, T) \cap \mathcal{F}_{K}(T) \neq \emptyset$.

In this work, we give new short proofs of Du-Karapinar-Shahzad's intersection theorems of $\mathcal{C O P} \mathcal{P}_{K}(g, T)$ and $\mathcal{F}_{K}(T)$ for multivalued non-self-maps (i.e., Theorems 1.1 and 1.2) by applying an existence theorem for approximate fixed point property.

\section{Some auxiliary key results}

Let $(X, d)$ be a metric space. Recall that a function $p: X \times X \rightarrow[0, \infty)$ is said to be a $\tau$-function $[3-5,7,8,20-22]$, first introduced and studied by Lin and $\mathrm{Du}$, if the following conditions hold:

( $\tau 1) p(x, z) \leq p(x, y)+p(y, z)$ for all $x, y, z \in X$

( $\tau 2)$ if $x \in X$ and $\left\{y_{n}\right\}$ in $X$ with $\lim _{n \rightarrow \infty} y_{n}=y$ such that $p\left(x, y_{n}\right) \leq M$ for some $M=M(x)>$ 0 , then $p(x, y) \leq M$;

( $\tau 3)$ for any sequence $\left\{x_{n}\right\}$ in $X$ with $\lim _{n \rightarrow \infty} \sup \left\{p\left(x_{n}, x_{m}\right): m>n\right\}=0$, if there exists a sequence $\left\{y_{n}\right\}$ in $X$ such that $\lim _{n \rightarrow \infty} p\left(x_{n}, y_{n}\right)=0$, then $\lim _{n \rightarrow \infty} d\left(x_{n}, y_{n}\right)=0$;

( $\tau 4)$ for $x, y, z \in X, p(x, y)=0$ and $p(x, z)=0$ imply $y=z$.

Note that with the additional condition

( $\tau 5) ~ p(x, x)=0$ for all $x \in X$,

a $\tau$-function becomes a $\tau^{0}$-function $[3-5,7,8]$ introduced by $\mathrm{Du}$. 
Clearly, any metric $d$ is a $\tau^{0}$-function. Observe further that if $p$ is a $\tau^{0}$-function, then, from $(\tau 4)$ and $(\tau 5), p(x, y)=0$ if and only if $x=y$.

Example A [7] Let $X=\mathbb{R}$ with the metric $d(x, y)=|x-y|$ and $0<a<b$. Define the function $p: X \times X \rightarrow[0, \infty)$ by

$$
p(x, y)=\max \{a(y-x), b(x-y)\}
$$

Then $p$ is nonsymmetric and hence $p$ is not a metric. It is easy to see that $p$ is a $\tau^{0}$-function.

Lemma 2.1 [22, Lemma 2.1] Let $(X, d)$ be a metric space and $p: X \times X \rightarrow[0, \infty)$ be a function. Assume that $p$ satisfies the condition ( $\tau 3)$. If a sequence $\left\{x_{n}\right\}$ in $X$ with $\lim _{n \rightarrow \infty} \sup \left\{p\left(x_{n}, x_{m}\right): m>n\right\}=0$, then $\left\{x_{n}\right\}$ is a Cauchy sequence in $X$.

Let $(X, d)$ be a metric space and $p$ be a $\tau$-function. A multivalued map $T: X \rightarrow \mathcal{N}(X)$ is said to have $p$-approximate fixed point property on $X$ provided

$$
\inf _{x \in X} p(x, T x)=0 .
$$

The following characterizations of $\mathcal{M T}$-functions proved first by $\mathrm{Du}$ [6] are quite useful for proving our main results.

Theorem 2.1 [6, Theorem 2.1] Let $\varphi:[0, \infty) \rightarrow[0,1)$ be a function. Then the following statements are equivalent.

(a) $\varphi$ is an $\mathcal{M T}$-function.

(b) For each $t \in[0, \infty)$, there exist $r_{t}^{(1)} \in[0,1)$ and $\varepsilon_{t}^{(1)}>0$ such that $\varphi(s) \leq r_{t}^{(1)}$ for all $s \in\left(t, t+\varepsilon_{t}^{(1)}\right)$.

(c) For each $t \in[0, \infty)$, there exist $r_{t}^{(2)} \in[0,1)$ and $\varepsilon_{t}^{(2)}>0$ such that $\varphi(s) \leq r_{t}^{(2)}$ for all $s \in\left[t, t+\varepsilon_{t}^{(2)}\right]$.

(d) For each $t \in[0, \infty)$, there exist $r_{t}^{(3)} \in[0,1)$ and $\varepsilon_{t}^{(3)}>0$ such that $\varphi(s) \leq r_{t}^{(3)}$ for all $s \in\left(t, t+\varepsilon_{t}^{(3)}\right]$.

(e) For each $t \in[0, \infty)$, there exist $r_{t}^{(4)} \in[0,1)$ and $\varepsilon_{t}^{(4)}>0$ such that $\varphi(s) \leq r_{t}^{(4)}$ for all $s \in\left[t, t+\varepsilon_{t}^{(4)}\right)$.

(f) For any nonincreasing sequence $\left\{x_{n}\right\}_{n \in \mathbb{N}}$ in $[0, \infty)$, we have $0 \leq \sup _{n \in \mathbb{N}} \varphi\left(x_{n}\right)<1$.

(g) $\varphi$ is a function of contractive factor; that is, for any strictly decreasing sequence $\left\{x_{n}\right\}_{n \in \mathbb{N}}$ in $[0, \infty)$, we have $0 \leq \sup _{n \in \mathbb{N}} \varphi\left(x_{n}\right)<1$.

The following result was essentially proved by Du et al. in [4], but we give the proof for the sake of completeness and the readers convenience.

Lemma 2.2 [4, Lemma 3.1] Let $(X, d)$ be a metric space, $p$ be a $\tau^{0}$-function and $T: X \rightarrow$ $\mathcal{N}(X)$ be a multivalued map. Then the following statements are equivalent.

(Q1) There exist a function $\xi:[0, \infty) \rightarrow[0, \infty)$ and an $\mathcal{M T}$-function $\varphi:[0, \infty) \rightarrow[0,1)$ such that for each $x \in X$, if $y \in$ Tx with $y \neq x$, then there exists $z \in$ Ty such that

$$
p(y, z) \leq \varphi(\xi(p(x, y))) p(x, y)
$$


(Q2) There exist a function $\tau:[0, \infty) \rightarrow[0, \infty)$ and an $\mathcal{M T}$-function $\kappa:[0, \infty) \rightarrow[0,1)$ such that for each $x \in X$,

$$
p(y, T y) \leq \kappa(\tau(p(x, y))) p(x, y) \quad \text { for all } y \in T x .
$$

Proof If (Q1) holds, then it is easy to verify that (Q2) also holds with $\kappa \equiv \varphi$ and $\tau \equiv \xi$. So it suffices to prove that ' $(\mathrm{Q} 2) \Rightarrow(\mathrm{Q} 1)$ '. Suppose that $(\mathrm{Q} 2)$ holds. Define $\varphi:[0, \infty) \rightarrow[0,1)$ by $\varphi(t)=\frac{1+\kappa(t)}{2}$. Then $\varphi$ is also an $\mathcal{M T}$-function. Indeed, it is obvious that $0 \leq \kappa(t)<\varphi(t)<1$ for all $t \in[0, \infty)$. Let $\left\{x_{n}\right\}_{n \in \mathbb{N}}$ be a strictly decreasing sequence in $[0, \infty)$. Since $\kappa$ is an $\mathcal{M T}$-function, by (g) of Theorem 2.1, we get

$$
0 \leq \sup _{n \in \mathbb{N}} \kappa\left(x_{n}\right)<1
$$

and hence

$$
0<\sup _{n \in \mathbb{N}} \varphi\left(x_{n}\right)=\frac{1}{2}\left[1+\sup _{n \in \mathbb{N}} \kappa\left(x_{n}\right)\right]<1 .
$$

So, by Theorem 2.1 again, we prove that $\varphi$ is an $\mathcal{M T}$-function.

For each $x \in X$, let $y \in T x$ with $y \neq x$. Then $p(x, y)>0$. By (Q2), we have

$$
p(y, T y)<\varphi(\tau(p(x, y))) p(x, y) .
$$

Since $\varphi(t)>0$ for all $t \in[0, \infty)$, there exists $z \in T y$ such that

$$
p(y, z)<\varphi(\tau(p(x, y))) p(x, y)
$$

which shows that (Q1) holds with $\xi \equiv \tau$. So, by above, we prove '(Q1) $\Longleftrightarrow(\mathrm{Q} 2)$ '.

Now, we present an existence theorem for $p$-approximate fixed point property and approximate fixed point property, which is indeed a somewhat generalized form of [4, Theorem 3.3] and is one of the key technical devices in the new short proofs of Theorems 1.1 and 1.2.

Theorem 2.2 Let $(X, d)$ be a metric space, $p$ be a $\tau^{0}$-function and $T: X \rightarrow \mathcal{N}(X)$ be a multivalued map. Assume that one of (L1) and (L2) is satisfied, where

(L1) there exist a nondecreasing function $\xi:[0, \infty) \rightarrow[0, \infty)$ and an $\mathcal{M T}$-function $\varphi:[0, \infty) \rightarrow[0,1)$ such that for each $x \in X$, if $y \in T x$ with $y \neq x$, then there exists $z \in$ Ty such that

$$
p(y, z) \leq \varphi(\xi(p(x, y))) p(x, y)
$$

(L2) there exist a nondecreasing function $\tau:[0, \infty) \rightarrow[0, \infty)$ and an $\mathcal{M T}$-function $\kappa:[0, \infty) \rightarrow[0,1)$ such that for each $x \in X$,

$$
p(y, T y) \leq \kappa(\tau(p(x, y))) p(x, y) \quad \text { for all } y \in T x
$$

Then the following statements hold. 
(a) There exists a Cauchy sequence $\left\{x_{n}\right\}_{n \in \mathbb{N}}$ in $X$ such that

(i) $x_{n+1} \in T x_{n}$ for all $n \in \mathbb{N}$,

(ii) $\inf _{n \in X} p\left(x_{n}, x_{n+1}\right)=\lim _{n \rightarrow \infty} p\left(x_{n}, x_{n+1}\right)=\lim _{n \rightarrow \infty} d\left(x_{n}, x_{n+1}\right)=\inf _{n \in \mathbb{N}} d\left(x_{n}\right.$, $\left.x_{n+1}\right)=0$.

(b) $\inf _{x \in X} p(x, T x)=\inf _{x \in X} d(x, T x)=0$; that is, $T$ has $p$-approximate fixed point property and approximate fixed point property on $X$.

Proof By Lemma 2.2, it suffices to prove that the conclusions hold under assumption (L1). Let $u \in X$ be given. If $u \in T u$, then

$$
\inf _{x \in X} p(x, T x) \leq p(u, T u) \leq p(u, u)=0,
$$

and

$$
\inf _{x \in X} d(x, T x) \leq d(u, u)=0
$$

which implies that $\inf _{x \in X} p(x, T x)=\inf _{x \in X} d(x, T x)=0$. Let $w_{n}=u$ for all $n \in \mathbb{N}$. Thus we have

$$
\begin{aligned}
& w_{n+1}=u \in T u=T w_{n} \quad \text { for all } n \in \mathbb{N}, \\
& \lim _{n \rightarrow \infty} p\left(w_{n}, w_{n+1}\right)=\inf _{n \in \mathbb{N}} p\left(w_{n}, w_{n+1}\right)=p(u, u)=0,
\end{aligned}
$$

and

$$
\lim _{n \rightarrow \infty} d\left(w_{n}, w_{n+1}\right)=\inf _{n \in \mathbb{N}} d\left(w_{n}, w_{n+1}\right)=d(u, u)=0 .
$$

Clearly,

$$
p\left(w_{n+1}, w_{n+2}\right)=0=\varphi\left(\xi\left(p\left(w_{n}, w_{n+1}\right)\right)\right) p\left(w_{n}, w_{n+1}\right) \quad \text { for all } n \in \mathbb{N}
$$

So, conclusions (a) and (b) hold in this case $u \in T u$, no matter what condition one begins with. Suppose that $u \notin T u$. Put $x_{1}=u$ and $x_{2} \in T x_{1}$. Then $x_{2} \neq x_{1}$ and hence $p\left(x_{1}, x_{2}\right)>0$. Assume that condition (L1) is satisfied. Then there exists $x_{3} \in T x_{2}$ such that

$$
p\left(x_{2}, x_{3}\right) \leq \varphi\left(\xi\left(p\left(x_{1}, x_{2}\right)\right)\right) p\left(x_{1}, x_{2}\right) .
$$

If $x_{2}=x_{3} \in T x_{2}$, then, following a similar argument as above, the conclusions are also proved. If $x_{3} \neq x_{2}$, then there exists $x_{4} \in T x_{3}$ such that

$$
p\left(x_{3}, x_{4}\right) \leq \varphi\left(\xi\left(p\left(x_{2}, x_{3}\right)\right)\right) p\left(x_{2}, x_{3}\right)
$$

By induction, we can obtain a sequence $\left\{x_{n}\right\}$ in $X$ satisfying $x_{n+1} \in T x_{n}$ and

$$
p\left(x_{n+1}, x_{n+2}\right) \leq \varphi\left(\xi\left(p\left(x_{n}, x_{n+1}\right)\right)\right) p\left(x_{n}, x_{n+1}\right) \quad \text { for all } n \in \mathbb{N} \text {. }
$$


Since $\varphi(t)<1$ for all $t \in[0, \infty)$, inequality (2.1) implies that the sequence $\left\{p\left(x_{n}, x_{n+1}\right)\right\}_{n \in \mathbb{N}}$ is strictly decreasing in $[0, \infty)$. Hence

$$
\lim _{n \rightarrow \infty} p\left(x_{n}, x_{n+1}\right)=\inf _{n \in \mathbb{N}} p\left(x_{n}, x_{n+1}\right) \geq 0 \quad \text { exists. }
$$

Since $\xi$ is nondecreasing, $\left\{\xi\left(p\left(x_{n}, x_{n+1}\right)\right)\right\}_{n \in \mathbb{N}}$ is a nonincreasing sequence in $[0, \infty)$. Since $\varphi$ is an $\mathcal{M T}$-function, by (g) of Theorem 2.1, we have

$$
0 \leq \sup _{n \in \mathbb{N}} \varphi\left(\xi\left(p\left(x_{n}, x_{n+1}\right)\right)\right)<1
$$

Let $\lambda:=\sup _{n \in \mathbb{N}} \varphi\left(\xi\left(p\left(x_{n}, x_{n+1}\right)\right)\right)$. So $\lambda \in[0,1)$ and we get from (2.1) that

$$
p\left(x_{n+1}, x_{n+2}\right) \leq \lambda p\left(x_{n}, x_{n+1}\right) \leq \cdots \leq \lambda^{n} p\left(x_{1}, x_{2}\right) \quad \text { for each } n \in \mathbb{N} \text {. }
$$

Since $\lambda \in[0,1), \lim _{n \rightarrow \infty} \lambda^{n}=0$ and hence the last inequality implies

$$
\lim _{n \rightarrow \infty} p\left(x_{n}, x_{n+1}\right)=0
$$

By (2.2) and (2.4), we obtain

$$
\inf _{n \in \mathbb{N}} p\left(x_{n}, x_{n+1}\right)=\lim _{n \rightarrow \infty} p\left(x_{n}, x_{n+1}\right)=0 .
$$

Now, we claim that $\left\{x_{n}\right\}$ is a Cauchy sequence in $X$. Let $\alpha_{n}=\frac{\lambda^{n-1}}{1-\lambda} p\left(x_{1}, x_{2}\right), n \in \mathbb{N}$. For $m, n \in$ $\mathbb{N}$ with $m>n$, by (2.3), we have

$$
p\left(x_{n}, x_{m}\right) \leq \sum_{j=n}^{m-1} p\left(x_{j}, x_{j+1}\right)<\alpha_{n} .
$$

Since $\lambda \in[0,1), \lim _{n \rightarrow \infty} \alpha_{n}=0$ and hence

$$
\lim _{n \rightarrow \infty} \sup \left\{p\left(x_{n}, x_{m}\right): m>n\right\}=0 .
$$

Applying Lemma 2.1, we show that $\left\{x_{n}\right\}$ is a Cauchy sequence in $X$. Hence $\lim _{n \rightarrow \infty} d\left(x_{n}\right.$, $\left.x_{n+1}\right)=0$. Since $\inf _{n \in \mathbb{N}} d\left(x_{n}, x_{n+1}\right) \leq d\left(x_{m}, x_{m+1}\right)$ for all $m \in \mathbb{N}$ and $\lim _{m \rightarrow \infty} d\left(x_{m}, x_{m+1}\right)=0$, one also obtains

$$
\lim _{n \rightarrow \infty} d\left(x_{n}, x_{n+1}\right)=\inf _{n \in \mathbb{N}} d\left(x_{n}, x_{n+1}\right)=0 .
$$

So conclusion (a) is proved. To see (b), since $x_{n+1} \in T x_{n}$ for each $n \in \mathbb{N}$, we have

$$
\inf _{x \in X} p(x, T x) \leq p\left(x_{n}, T x_{n}\right) \leq p\left(x_{n}, f x_{n+1}\right)
$$

and

$$
\inf _{x \in X} d(x, T x) \leq d\left(x_{n}, T x_{n}\right) \leq d\left(x_{n}, f x_{n+1}\right)
$$


for all $n \in \mathbb{N}$. Combining (2.6), (2.7) and (2.8), we get

$$
\inf _{x \in X} p(x, T x)=\inf _{x \in X} d(x, T x)=0 .
$$

The proof is completed.

The following existence theorem is obviously an immediate result from Theorem 2.2.

Theorem 2.3 Let $(X, d)$ be a metric space, $p$ be a $\tau^{0}$-function and $T: X \rightarrow \mathcal{N}(X)$ be a multivalued map. Assume that one of $(\mathrm{H} 1)$ and $(\mathrm{H} 2)$ is satisfied, where

(H1) there exists an $\mathcal{M T}$-function $\alpha:[0, \infty) \rightarrow[0,1)$ such that for each $x \in X$, if $y \in T x$ with $y \neq x$, then there exists $z \in$ Ty such that

$$
p(y, z) \leq \alpha(p(x, y)) p(x, y)
$$

(H2) there exists an $\mathcal{M T}$-function $\beta:[0, \infty) \rightarrow[0,1)$ such that for each $x \in X$,

$$
p(y, T y) \leq \beta(p(x, y)) p(x, y) \quad \text { for all } y \in T x .
$$

Then the following statements hold.

(a) There exists a Cauchy sequence $\left\{x_{n}\right\}_{n \in \mathbb{N}}$ in $X$ such that

(i) $x_{n+1} \in T x_{n}$ for all $n \in \mathbb{N}$,

(ii) $\inf _{n \in X} p\left(x_{n}, x_{n+1}\right)=\lim _{n \rightarrow \infty} p\left(x_{n}, x_{n+1}\right)=\lim _{n \rightarrow \infty} d\left(x_{n}, x_{n+1}\right)=\inf _{n \in \mathbb{N}} d\left(x_{n}\right.$, $\left.x_{n+1}\right)=0$.

(b) $\inf _{x \in X} p(x, T x)=\inf _{x \in X} d(x, T x)=0$; that is, T has $p$-approximate fixed point property and approximate fixed point property on $X$.

Lemma 2.3 Let $\tau:[0, \infty) \rightarrow[0, \infty)$ be a nondecreasing function and $\kappa:[0, \infty) \rightarrow[0,1)$ be an $\mathcal{M T}$-function. Then $\kappa \circ \tau$ is an $\mathcal{M T}$-function.

Proof Let $\left\{x_{n}\right\}_{n \in \mathbb{N}}$ be a strictly decreasing sequence in $[0, \infty)$. Since $\tau$ is a nondecreasing function, $\left\{\tau\left(x_{n}\right)\right\}_{n \in \mathbb{N}}$ is a nonincreasing sequence in $[0, \infty)$. Since $\kappa$ is an $\mathcal{M T}$-function, by (f) of Theorem 2.1, we get

$$
0 \leq \sup _{n \in \mathbb{N}} \kappa\left(\tau\left(x_{n}\right)\right)<1
$$

or, equivalently,

$$
0 \leq \sup _{n \in \mathbb{N}}(\kappa \circ \tau)\left(x_{n}\right)<1 .
$$

So, by Theorem 2.1 again, we prove that $\kappa \circ \tau$ is an $\mathcal{M T}$-function.

Applying Lemma 2.3, we conclude that Theorem 2.2 is also a special case of Theorem 2.3. Therefore we obtain the following important fact.

Theorem 2.4 Theorem 2.2 and Theorem 2.3 are equivalent. 


\section{Short proofs of Theorems $\mathbf{1 . 1}$ and $\mathbf{1 . 2}$}

Let us see how we can utilize Theorem 2.3 to prove Theorem 1.1.

Short proof of Theorem 1.1 Since $K$ is a nonempty closed subset of $X$ and $X$ is complete, $(K, d)$ is also a complete metric space. Let $x \in K$. Put $k=\frac{\gamma}{1-\gamma}$ and $\lambda=\frac{1+k}{2}$. So, $0 \leq k<\lambda<1$. Let $y \in T x \cap K$ be arbitrary. So, $d(y, T x \cap K)=0$. By (D2), we have $d(g y, T x \cap K)=0$. Hence inequality (1.1) implies

$$
\mathcal{H}(T x, T y \cap K) \leq \gamma[d(x, T x \cap K)+\mathcal{H}(T x, T y \cap K)] \quad \text { for all } y \in T x \cap K
$$

Inequality (3.1) shows that

$$
d(y, T y \cap K) \leq \mathcal{H}(T x, T y \cap K) \leq k d(x, T x \cap K)<\lambda d(x, y) \quad \text { for all } y \in T x \cap K
$$

Define $G: K \rightarrow \mathcal{C B}(K)$ by

$$
G x=T x \cap K \quad \text { for all } x \in K,
$$

and let $\mu:[0, \infty) \rightarrow[0,1)$ be defined by

$$
\eta(t)=\lambda \quad \text { for all } t \in[0, \infty)
$$

Then $\mu$ is an $\mathcal{M T}$-function. By (3.2), we obtain

$$
d(y, G y) \leq \mu(d(x, y)) d(x, y) \text { for all } y \in G x .
$$

Applying Theorem 2.3 with $p \equiv d$, there exists a Cauchy sequence $\left\{x_{n}\right\}_{n \in \mathbb{N}}$ in $K$ such that

$$
x_{n+1} \in G x_{n}=T x_{n} \cap K \quad \text { for all } n \in \mathbb{N}
$$

and

$$
\lim _{n \rightarrow \infty} d\left(x_{n}, x_{n+1}\right)=\inf _{n \in \mathbb{N}} d\left(x_{n}, x_{n+1}\right)=0 .
$$

By the completeness of $K$, there exists $v \in K$ such that $x_{n} \rightarrow v$ as $n \rightarrow \infty$. By (3.3) and (D2), we have

$$
g x_{n+1} \in T x_{n} \cap K \quad \text { for each } n \in \mathbb{N} \text {. }
$$

Since $g$ is continuous and $\lim _{n \rightarrow \infty} x_{n}=v$, we have

$$
\lim _{n \rightarrow \infty} g x_{n}=g \nu
$$

Since the function $x \mapsto d(x, T v)$ is continuous, by (1.1), (3.3), (3.4), (3.5) and (3.6), we get

$$
\begin{aligned}
d(v, T v \cap K) & =\lim _{n \rightarrow \infty} d\left(x_{n+1}, T v \cap K\right) \\
& \leq \lim _{n \rightarrow \infty} \mathcal{H}\left(T x_{n}, T v \cap K\right)
\end{aligned}
$$




$$
\begin{aligned}
\leq & \lim _{n \rightarrow \infty}\left\{\gamma\left[d\left(x_{n}, T x_{n} \cap K\right)+d\left(v, T x_{n} \cap K\right)+d(v, T v \cap K)\right]\right. \\
& \left.+h(v) d\left(g v, T x_{n} \cap K\right)\right\} \\
\leq & \lim _{n \rightarrow \infty}\left\{\gamma\left[d\left(x_{n}, x_{n+1}\right)+d\left(v, x_{n+1}\right)+d(v, T v \cap K)\right]+h(v) d\left(g v, g x_{n+1}\right)\right\} \\
= & \gamma d(v, T v \cap K),
\end{aligned}
$$

which implies $d(v, T v \cap K)=0$. By the closedness of $T v$, we have $v \in T v \cap K$. From (D2), $g v \in T v \cap K \subseteq T v$. Hence we verify $v \in \mathcal{C O P}_{K}(g, T) \cap \mathcal{F}_{K}(T)$. The proof is complete.

In order to finish off our work, let us prove Theorem 1.2 by applying Theorem 2.3.

Short proof of Theorem 1.2 Since $K$ is a nonempty closed subset of $X$ and $X$ is complete, $(K, d)$ is also a complete metric space. Note first that for each $x \in K$, by (D2), we have $d(g y, T x \cap K)=0$ for all $y \in T x \cap K$. So, for each $x \in K$, by (1.2), we obtain

$$
d(y, T y \cap K) \leq \varphi(d(x, y)) d(x, y) \quad \text { for all } y \in T x \cap K .
$$

Define $G: K \rightarrow \mathcal{C B}(K)$ by

$$
G x=T x \cap K \quad \text { for all } x \in K \text {. }
$$

From (3.7), we obtain

$$
d(y, G y) \leq \varphi(d(x, y)) d(x, y) \quad \text { for all } y \in G x .
$$

By using Theorem 2.3, there exists a Cauchy sequence $\left\{x_{n}\right\}_{n \in \mathbb{N}}$ in $K$ such that

$$
x_{n+1} \in G x_{n}=T x_{n} \cap K \quad \text { for all } n \in \mathbb{N}
$$

and

$$
\lim _{n \rightarrow \infty} d\left(x_{n}, x_{n+1}\right)=\inf _{n \in \mathbb{N}} d\left(x_{n}, x_{n+1}\right)=0 .
$$

By the completeness of $K$, there exists $v \in K$ such that $x_{n} \rightarrow v$ as $n \rightarrow \infty$. Thanks to (3.8) and (D2), we have

$$
g x_{n+1} \in T x_{n} \cap K \quad \text { for each } n \in \mathbb{N} \text {. }
$$

Since $g$ is continuous and $\lim _{n \rightarrow \infty} x_{n}=v$, we have

$$
\lim _{n \rightarrow \infty} g x_{n}=g \nu
$$

Since the function $x \mapsto d(x, T v)$ is continuous, by (1.2), (3.8), (3.10) and (3.11), we get

$$
\begin{aligned}
d(v, T v \cap K) & =\lim _{n \rightarrow \infty} d\left(x_{n+1}, T v \cap K\right) \\
& \leq \lim _{n \rightarrow \infty} \mathcal{H}\left(T x_{n}, T v \cap K\right)
\end{aligned}
$$




$$
\begin{aligned}
& \leq \lim _{n \rightarrow \infty}\left\{\varphi\left(d\left(x_{n}, v\right)\right) d\left(x_{n}, v\right)+h(v) d\left(g v, T x_{n} \cap K\right)\right\} \\
& \leq \lim _{n \rightarrow \infty}\left\{\varphi\left(d\left(x_{n}, v\right)\right) d\left(x_{n}, v\right)+h(v) d\left(g v, g x_{n+1}\right)\right\}=0,
\end{aligned}
$$

which implies $d(v, T v \cap K)=0$. By the closedness of $T v$, we have $v \in T v \cap K$. By (D2), $g v \in T v \cap K \subseteq T v$ and hence $v \in \mathcal{C O P}_{K}(g, T) \cap \mathcal{F}_{K}(T)$. The proof is complete.

\section{Competing interests}

The author declares that he has no competing interests.

\section{Acknowledgements}

In this research, the author was supported by grant No. NSC 102-2115-M-017-001 of the National Science Council of the Republic of China.

\section{Received: 30 July 2013 Accepted: 24 September 2013 Published: 08 Nov 2013}

\section{References}

1. Hussain, N, Amini-Harandi, A, Cho, YJ: Approximate endpoints for set-valued contractions in metric spaces. Fixed Point Theory Appl. 2010, Article ID 614867 (2010). doi:10.1155/2010/614867

2. Khamsi, MA: On asymptotically nonexpansive mappings in hyperconvex metric spaces. Proc. Am. Math. Soc. 132, 365-373 (2004)

3. Du, W-S: On approximate coincidence point properties and their applications to fixed point theory. J. Appl. Math. 2012, Article ID 302830 (2012). doi:10.1155/2012/302830

4. Du, W-S, He, Z, Chen, Y-L: New existence theorems for approximate coincidence point property and approximate fixed point property with applications to metric fixed point theory. J. Nonlinear Convex Anal. 13(3), 459-474 (2012)

5. Du, W-S: New existence results and generalizations for coincidence points and fixed points without global completeness. Abstr. Appl. Anal. 2013, Article ID 214230 (2013). doi:10.1155/2013/214230

6. Du, W-S: On coincidence point and fixed point theorems for nonlinear multivalued maps. Topol. Appl. 159, 49-56 (2012)

7. Du, W-S: Some new results and generalizations in metric fixed point theory. Nonlinear Anal. 73, 1439-1446 (2010)

8. Du, W-S: On generalized weakly directional contractions and approximate fixed point property with applications. Fixed Point Theory Appl. 2012, 6 (2012). doi:10.1186/1687-1812-2012-6

9. Sintunavarat, W, Kumam, P: Common fixed point theorems for hybrid generalized multi-valued contraction mappings. Appl. Math. Lett. 25(1), 52-57 (2012)

10. Kumam, P, Aydi, H, Karapinar, E, Sintunavarat, W: Best proximity points and extension of Mizoguchi-Takahashi's fixed point theorems. Fixed Point Theory Appl. (in press)

11. Du, W-S, Karapinar, E, Shahzad, N: The study of fixed point theory for various multivalued non-self-maps. Abstr. Appl. Anal. 2013, Article ID 938724 (2013). doi:10.1155/2013/938724

12. Browder, FE: Nonexpansive nonlinear operators in Banach space. Proc. Natl. Acad. Sci. USA 54, 1041-1044 (1965)

13. Browder, FE, Petryshyn, WV: Construction of fixed points of nonlinear mappings in Hilbert space. J. Math. Anal. Appl. 20, 197-228 (1967)

14. Kirk, WA: Remarks on pseudo-contractive map. Proc. Am. Math. Soc. 25, 820-823 (1970)

15. Kirk, WA: Fixed point theorems for nonlinear nonexpansive and generalized contraction mappings. Pac. J. Math. 38 89-94 (1971)

16. Assad, NA, Kirk, WA: Fixed point theorems for set-valued mappings of contractive type. Pac. J. Math. 43, 553-562 (1972)

17. Reich, S: Fixed points of condensing functions. J. Math. Anal. Appl. 41, 460-467 (1973)

18. Assad, NA: A fixed point theorem for some non-self-mappings. Tamkang J. Math. 21(4), 387-393 (1990)

19. Alghamdi, MA, Berinde, $V$, Shahzad, N: Fixed points of multivalued nonself almost contractions. J. Appl. Math. 2013, Article ID 621614 (2013). doi:10.1155/2013/621614

20. Lin, L-J, Du, W-S: Ekeland's variational principle, minimax theorems and existence of nonconvex equilibria in complete metric spaces. J. Math. Anal. Appl. 323, 360-370 (2006)

21. Lin, L-J, Du, W-S: On maximal element theorems, variants of Ekeland's variational principle and their applications. Nonlinear Anal. 68, 1246-1262 (2008)

22. Du, W-S: Critical point theorems for nonlinear dynamical systems and their applications. Fixed Point Theory Appl. 2010, Article ID 246382 (2010). doi:10.1155/2010/246382

10.1186/1029-242X-2013-506

Cite this article as: Du: A note on approximate fixed point property and Du-Karapinar-Shahzad's intersection theorems. Journal of Inequalities and Applications 2013, 2013:506 